\title{
Correction: Complexity theory for the modern Chinese economy from an information entropy perspective: Modeling of economic efficiency and growth potential
}

\author{
Jun Yan, Lianyong Feng, Artem Denisov, Alina Steblyanskaya, Jan-Pieter Oosterom
}

There is an error in affiliation 4 for author Alina Steblyanskaya. The correct affiliation 4 is: Laboratory of Microanalysis and Modeling, Central Economics and Mathematics Institute, Russian Academy of Science, Moscow, Russia.

In Fig 3, the Chinese providence "Shaanxi" is misspelled. The providence "Shan'xi" should be "Shaanxi." Please see the correct Fig 3 here.

G OPEN ACCESS

Citation: Yan J, Feng L, Denisov A, Steblyanskaya A, Oosterom J-P (2020) Correction: Complexity theory for the modern Chinese economy from an information entropy perspective: Modeling of economic efficiency and growth potential. PLoS ONE 15(3): e0230165. https://doi.org/10.1371/ journal.pone.0230165

Published: March 3, 2020

Copyright: $\odot 2020$ Yan et al. This is an open access article distributed under the terms of the Creative Commons Attribution License, which permits unrestricted use, distribution, and reproduction in any medium, provided the original author and source are credited. 
Flows (sum of input and output flow)

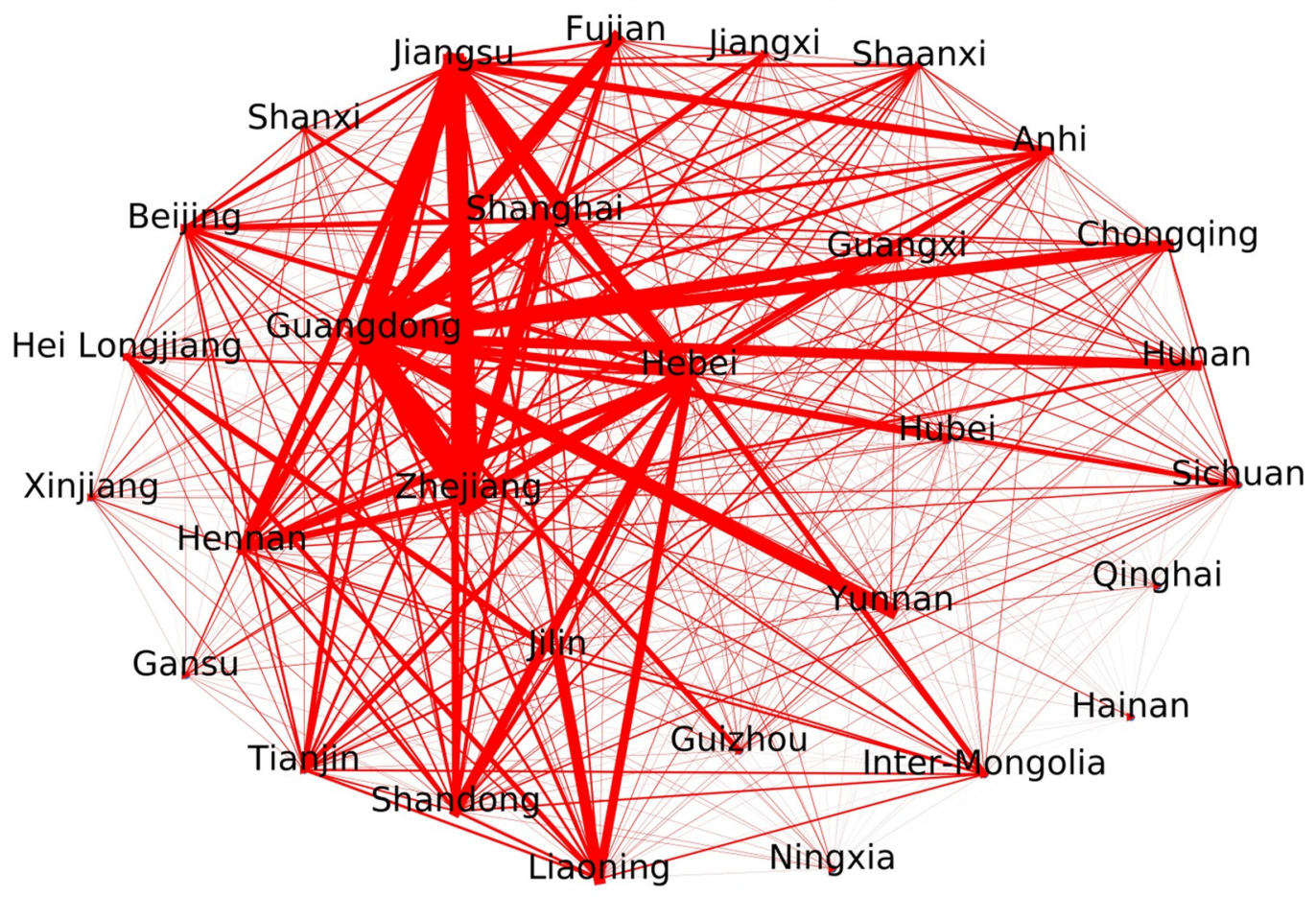

A)

Flows (sum of input and output flow)

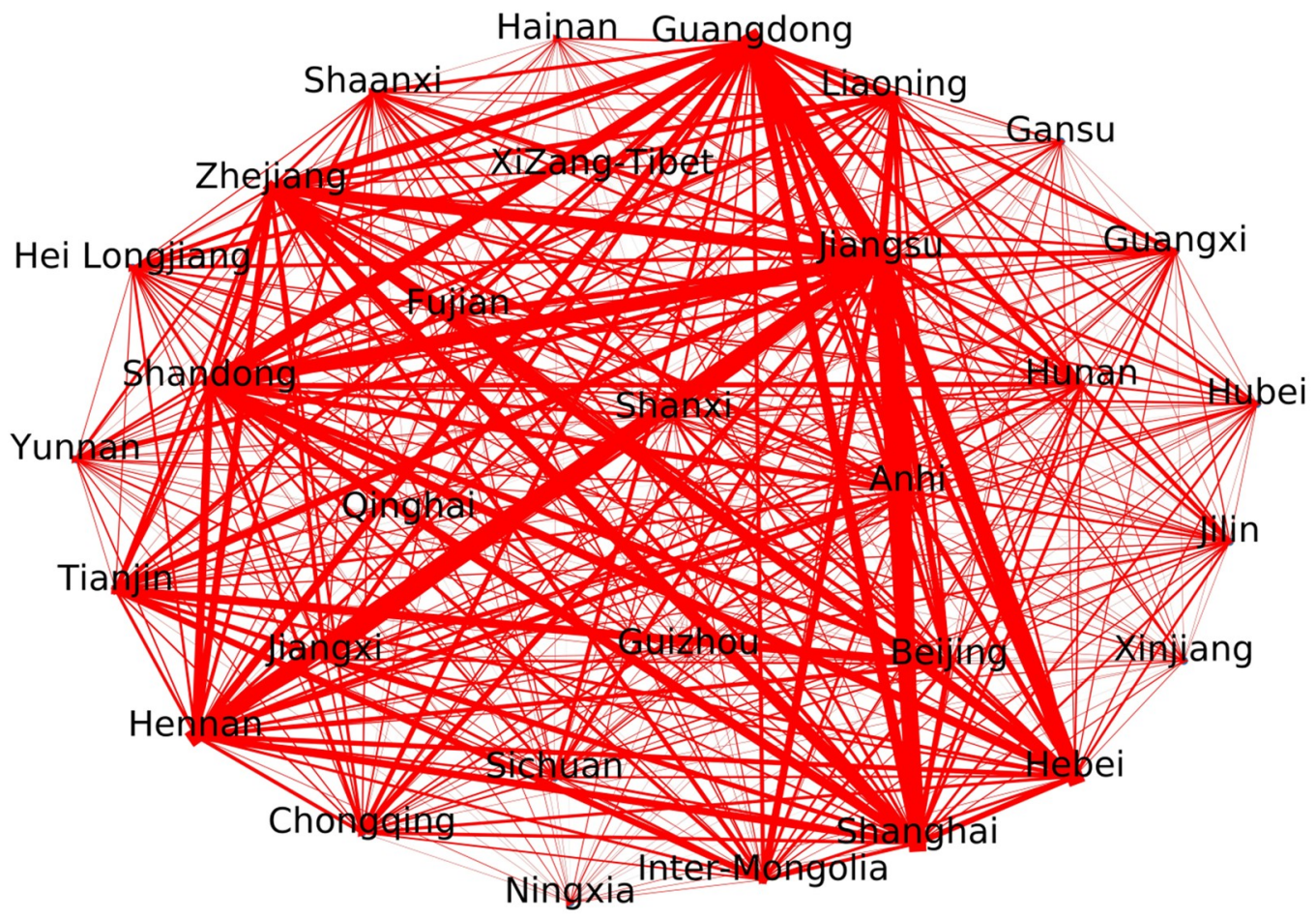

B)

Fig 3. A) 2007 flows between China Provinces B) 2012 flows between China Provinces.

https://doi.org/10.1371/journal.pone.0230165.g001 


\section{PLOS ONE}

\section{Reference}

1. Yan J, Feng L, Denisov A, Steblyanskaya A, Oosterom J-P (2020) Complexity theory for the modern Chinese economy from an information entropy perspective: Modeling of economic efficiency and growth potential. PLoS ONE 15(1): e0227206. https://doi.org/10.1371/journal.pone.0227206 PMID: 31990933 